\title{
Observations on the retinal pigment epithelium and retinal macrophages in experimental retinal detachment
}

\author{
N. F. JOHNSON AND W. S. FOULDS \\ From the Tennent Institute of Ophthalmology, Glasgow University and Western Infirmary, Glasgow
}

SUMMARY After experimental retinal detachment in rabbits macrophages are a prominent feature in the subretinal space or within the retina. Two sources for these macrophages are identified. The retinal pigment epithelium (RPE) may undergo metaplasia and actively 'bud'; the evolving macrophage is then formed by a vitreal protrusion of the cytoplasm of an RPE cell which is 'nipped off' by lateral protrusions from adjacent cells. In addition, in regions of RPE proliferation, bloodborne cells were found in Bruch's membrane and among the mass of proliferated RPE cells, suggesting that blood-borne cells may pass from the choroidal circulation through Bruch's membrane, and the RPE layer.

A distressing and still relatively unexplained complication of retinal detachment is the occasional occurrence after apparently successful surgery of gross fibrotic changes in the retina and vitreous leading to the clinical picture of massive vitreous retraction, or massive periretinal proliferation as it has been more recently termed (Machemer and Laqua, 1975). It has been suggested that cells originating from pigment epithelium may play an important role in the formation of periretinal membranes. It has been shown that intravitreal autotransplants of retinal pigment epithelium (RPE) may transform to cells having many of the characteristics of macrophages seen in experimental retinal detachment (Mandelcorn et al., 1975). But the mechanisms underlying the formation of these macrophages are speculative.

During an investigation into the distribution of acid mucopolysaccharides in detached retinae (Johnson and Foulds, 1976) it became apparent that the retinal pigment epithelium may be actively involved in the formation of macrophages. This paper describes the possible roles of the RPE in the formation of such cells.

\section{Material and Methods}

For this investigation 7 adult Dutch rabbits weighing between 1.6 and $2.2 \mathrm{~kg}$ were used. Detachment of the

Address for reprints: Dr N. F. Johnson, Tennent Institute of Ophthalmology, Western Infirmary, Glasgow G11 6NT retina was produced by a modification of the technique reported by Foulds (1963). The animals were anaesthetised with intraperitoneal pentobarbital (Nembutal), $60 \mathrm{mg} / \mathrm{kg}$. The pupil of one eye of each animal was maximally dilated with several drops of $1.0 \%$ cyclopentolate and $10 \%$ phenylephrine. The collagen framework of the posterior cortical vitreous was destroyed by the rotation of a blunted 23 gauge needle introduced into the vitreous cavity via the pars plana under direct vision through a contact lens with an operating microscope. In contrast to the original technique no hyaluronidase was injected. A hole in the retina was created by suction through the needle, and this resulted in a subtotal detachment of the retina. The other eye remained normal and acted as the control. Two animals were killed after 1 week, 1 after 2 weeks, and 4 animals after 3 weeks. The animals were killed by an overdose of pentobarbital. The eyes were enucleated and bisected in the equatorial plane; the posterior halves were immersed in fixative at room temperature. The fixative was $3 \%$ glutaraldehyde buffered in Sorensen's phosphate buffer (pH 7.3). Tissue was taken from the attached and detached areas of the test eyes and corresponding positions in the control eye and conventionally processed for electron microscopy. The tissue was embedded in Spurr's resin. Sections $1 \mu \mathrm{m}$ thick were stained with Loeffler's methylene blue for light microscopy. Sections $50-70 \mathrm{~nm}$ thick were stained with uranyl acetate and lead citrate and examined with a Philips 301 electron microscope. 


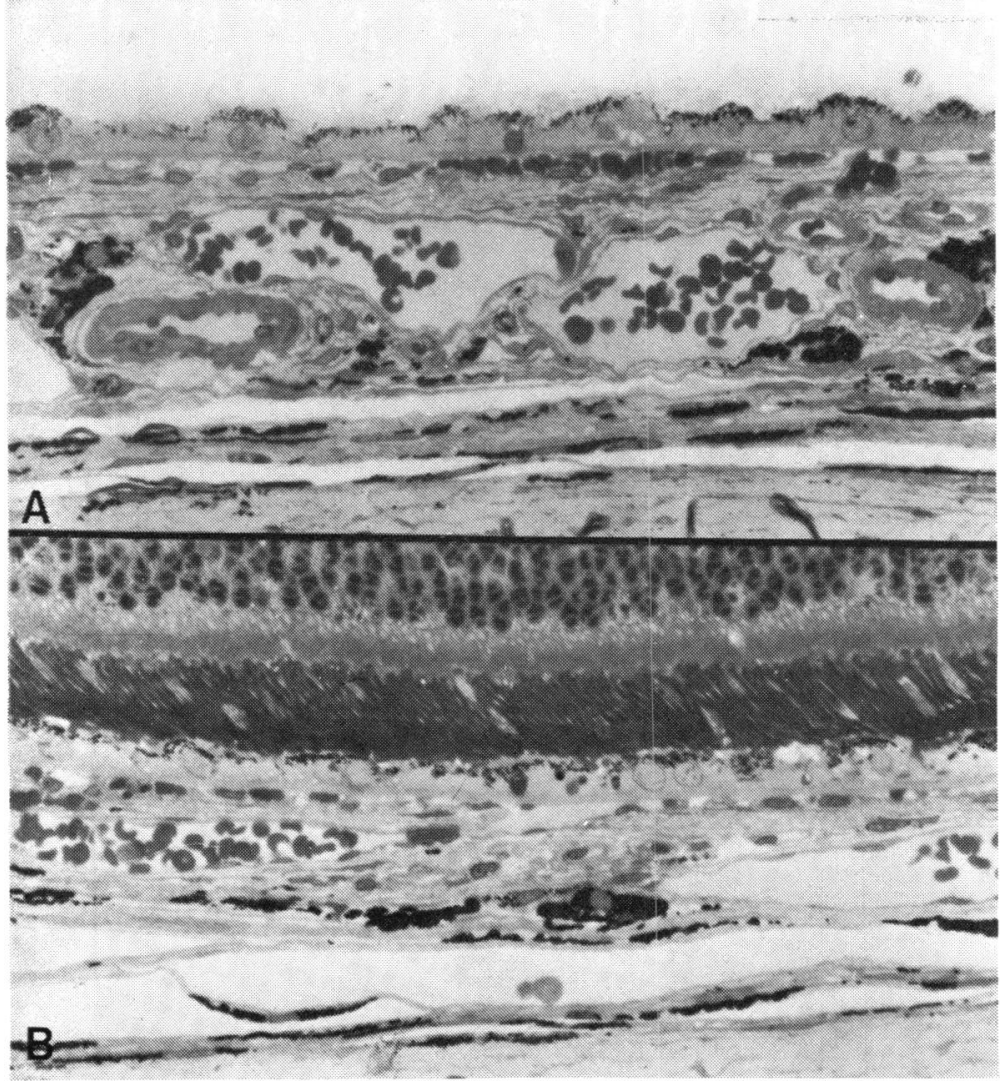

Fig. 1 Appearance of pigment epithelium under detached retina (A) and attached retina (B). In the areas shown the pigment epithelium under the detached retina retains a normal appearance $\times 640$

\section{Results}

There were no discernible differences in the appearance of the pigment epithelium from tissues taken at 1 week, 2 weeks, or 3 weeks after the production of a retinal detachment.

Examination of the pigment epithelium in areas where the retina was detached showed considerable variation in the appearance of the RPE in the same eye. In some regions the RPE under the detached retina retained a near normal appearance, which by light microscopy did not differ from RPE under normal attached retina (Fig. 1). By electron microscopy only slight ultrastructural changes were seen in these near-normal areas of pigment epithelium (Fig. 2). The apical processes were disorganised and more numerous than in the control tissue. Unlike normal RPE, where the mitochondria are found predominantly adjacent to the basal infoldings, the mitochondria were randomly distributed throughout the cytoplasm. The cytoplasm was generally devoid of phagosomes containing recognisable outer segment material. The cells completely covered Bruch's mem- brane, and the lateral junctional complexes appeared to be intact. These findings are similar to those described in the owl monkey (Kroll and Machemer, 1968), in the rabbit (Ohkuma, 1973), in the rat (Reich-d'Almeida and Hockley, 1975) and after secondary detachments in man (Kroll, 1969).

In other areas, although the pigment epithelium remained as a single layer, the individual cells showed gross abnormality with a vitreal displacement of the cytoplasm (Fig. 3). In addition, free pigmented cells were seen adjacent to the apical surface of the RPE layer. This considerable disturbance in the RPE was well seen on electron microscopy. In the areas being considered Bruch's membrane remained covered by RPE, but the individual RPE cells were affected by a cytoplasmic displacement which took a variety of forms; these could be placed into a sequence which may represent the formation of free macrophages from the RPE (Figs. 4-7).

The initial change from the flat uniform RPE cell was an alteration in cell shape. The apical surface of an RPE cell became convex (Fig. 4). This convexity became more marked (Fig. 5), and two ad- 


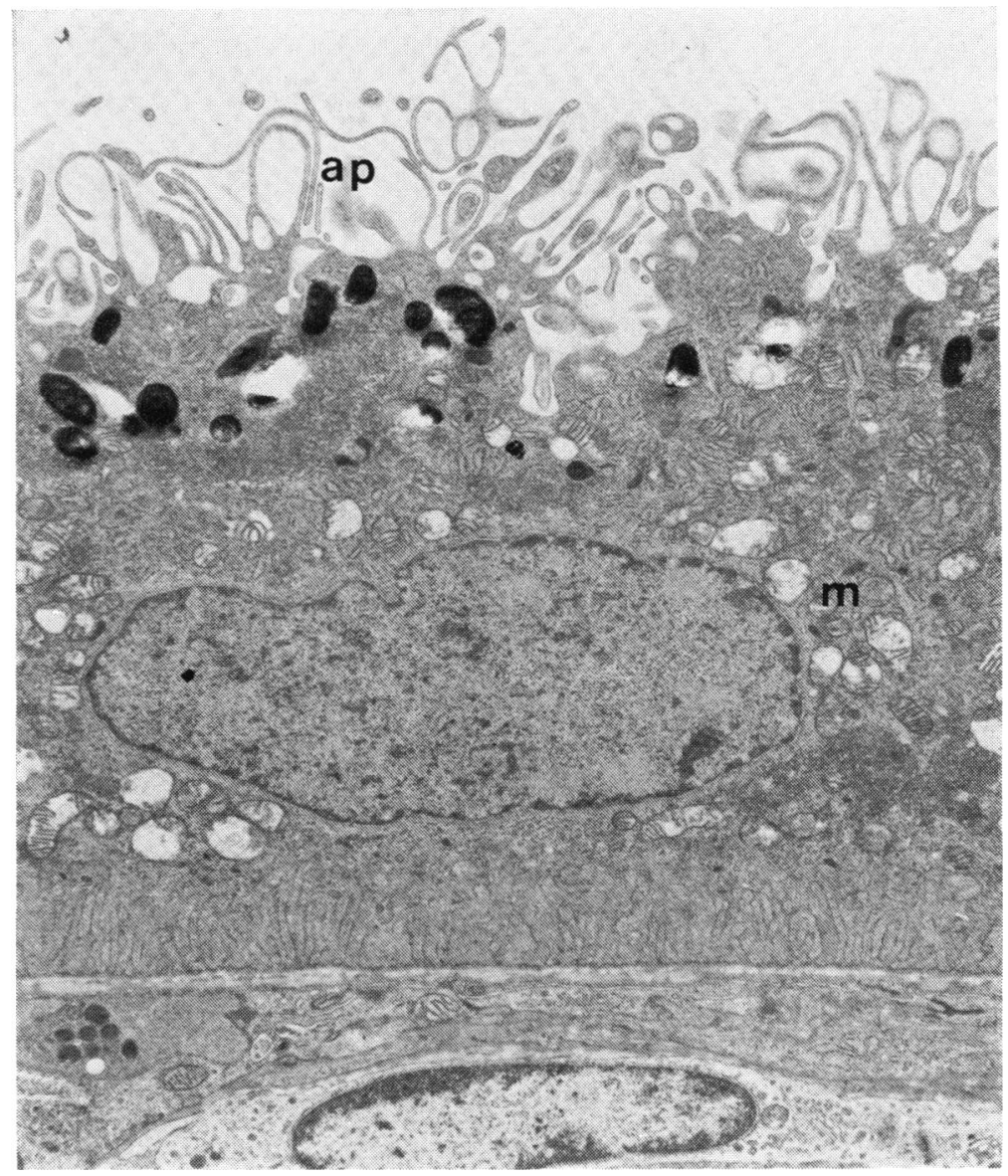

Fig. 2 Electron micrograph from same area as shown in Fig. 1A. The mitochondria (M) are randomly distributed throughout the cytoplasm. The apical processes (AP) are more numerous than normal $\times 5570$

jacent RPE cells pushed out lateral cytoplasmic protrusions which pinched in the basal part of the vitreally displaced cytoplasm (Fig. 6). Eventually the lateral processes of the adjacent cells 'nipped off' the remaining cytoplasm and resulted in the formation of a free cell, leaving the remaining portion of the cell cytoplasm still attached to Bruch's membrane (Fig. 7). The vitreally displaced cytoplasm and the free cell generally possessed a nucleus in every cell section studied. In these regions the lateral cell junctions were often more complicated than normal with the occurrence of a larger number of adhering type junctions. The free cells often contained melanin granules and had many characteristics of the underlying RPE. On occasion, however, some of the free cells had a lower cytoplasmic electron density than the RPE, and the pigment granules were confined to what appeared to be a secondary lysosomal complex 

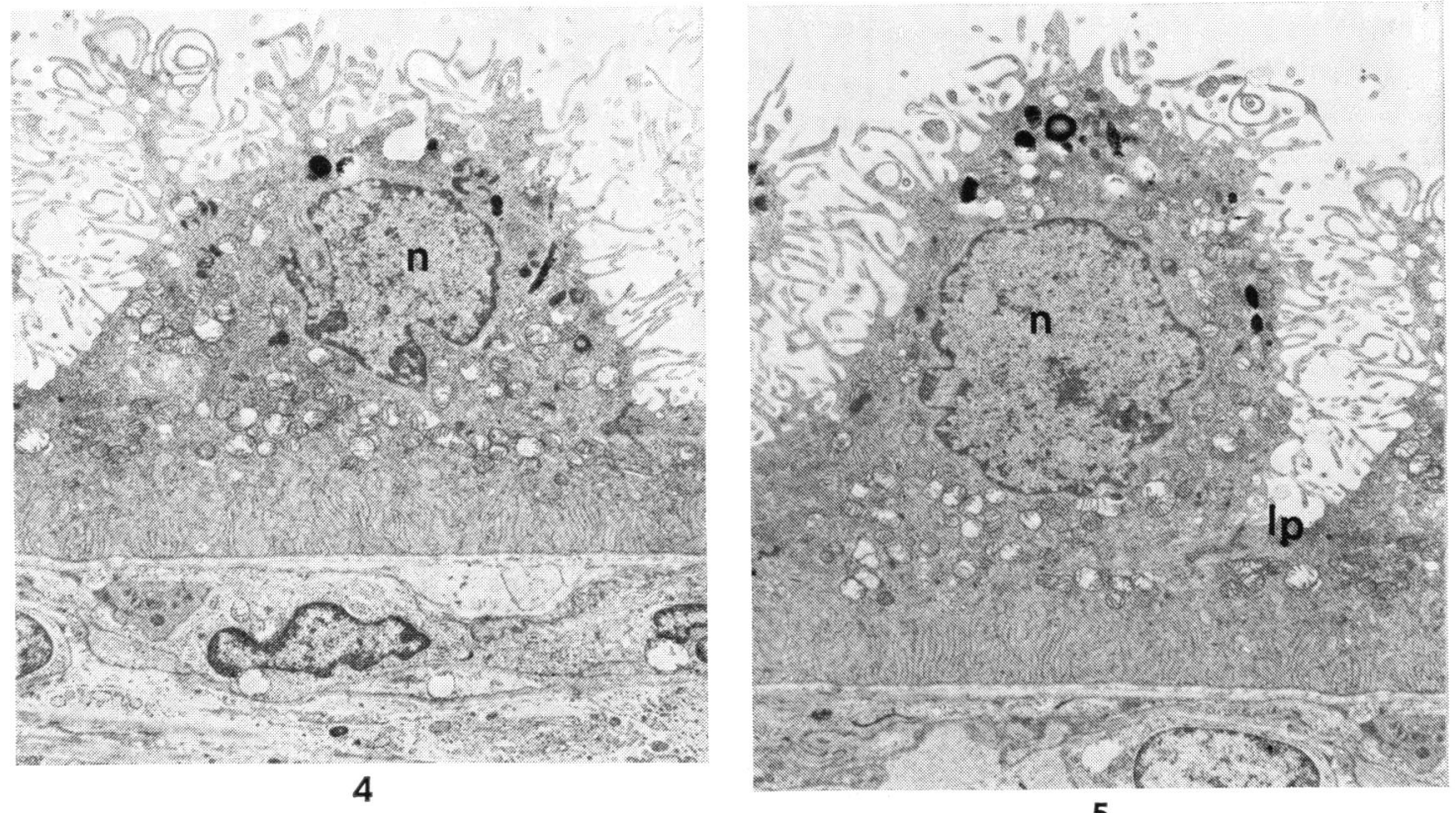

5

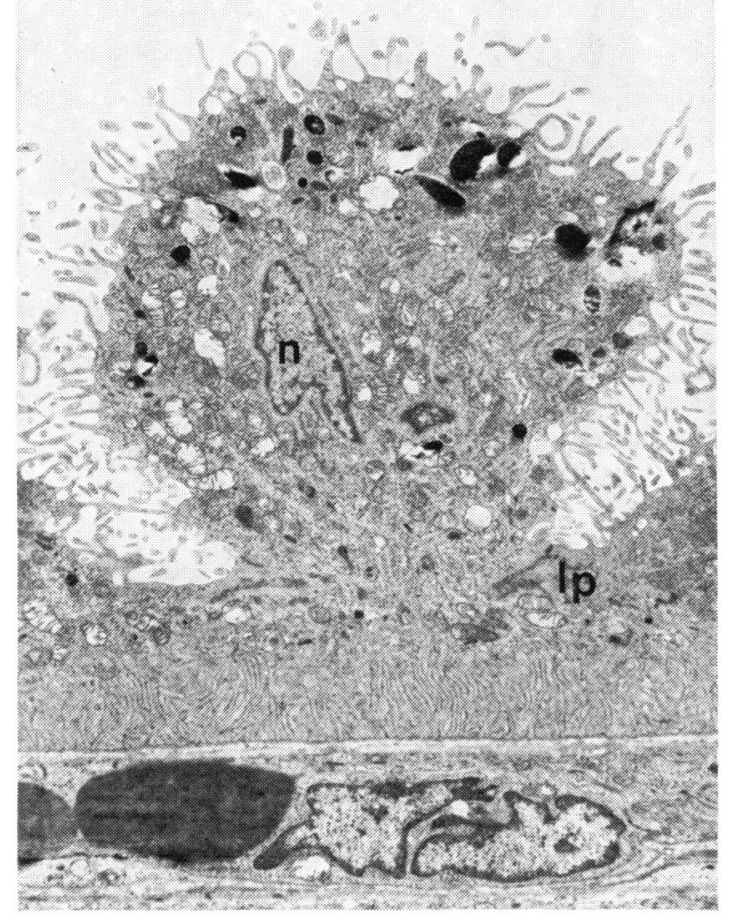

6

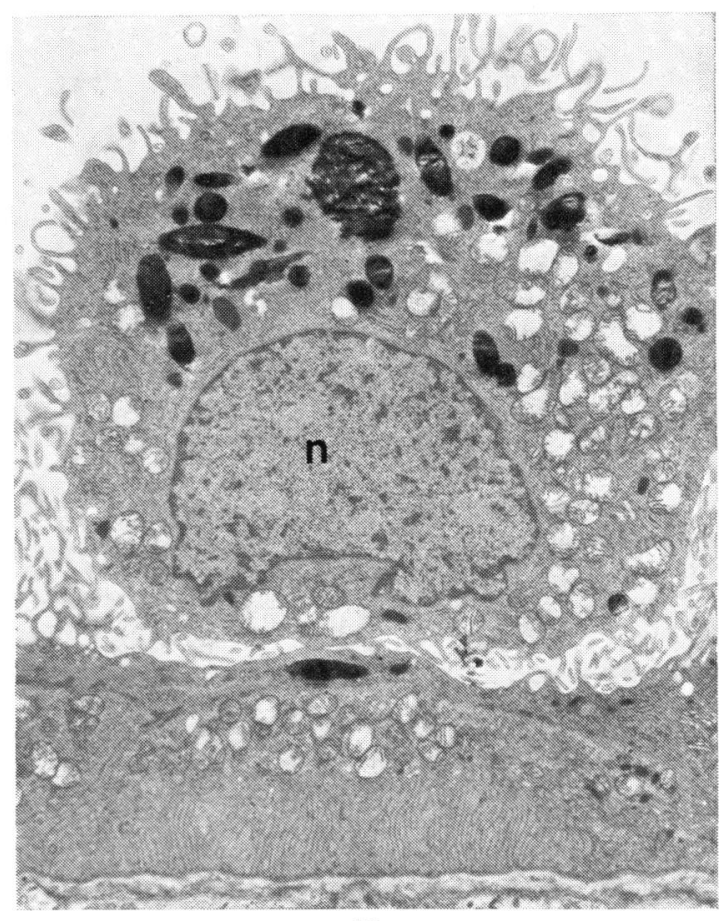

7

Figs. 4-7 Electron micrograph of changes occurring in the pigment epithelium under detached retina which may represent a sequence of events leading to the formation of free macrophages. $\mathrm{N}=$ pigment epithelial cell nucleus. $\mathrm{LP}=$ Lateral processes of neighbouring pigment epithelial cells $\times 5260$ 


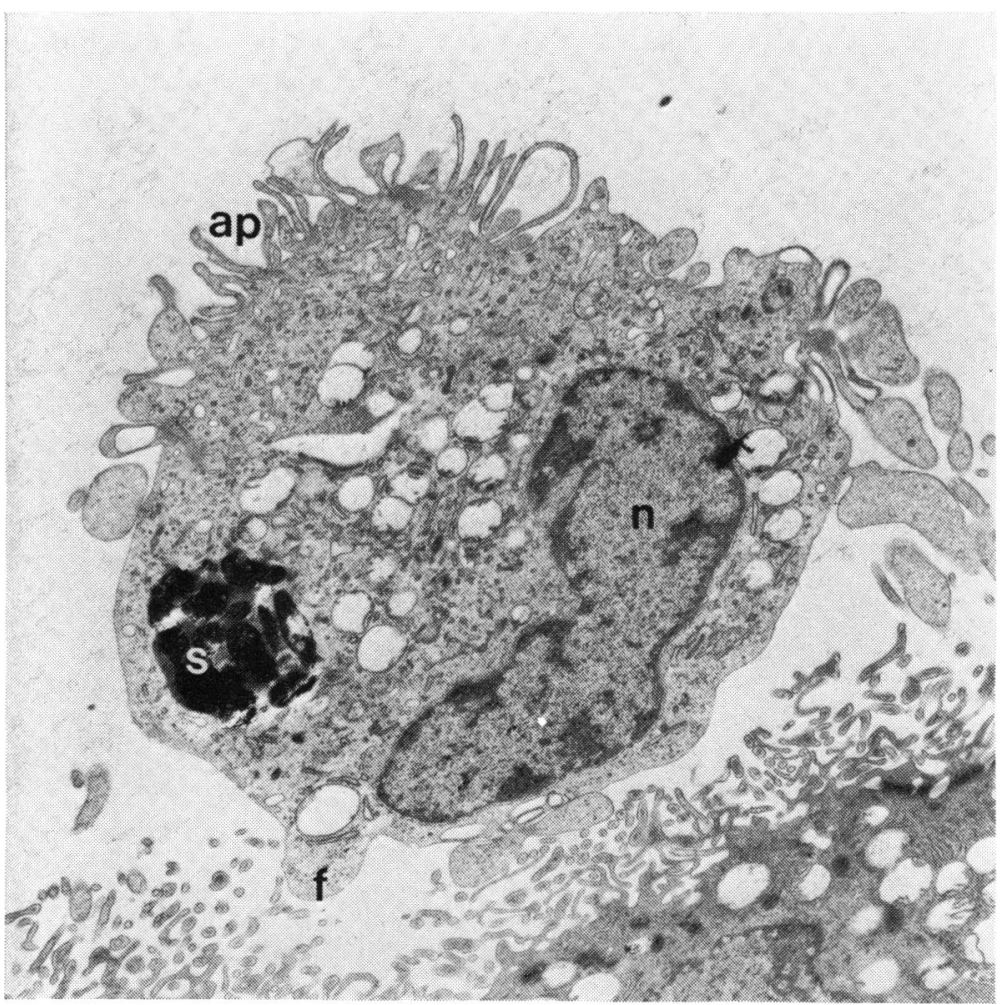

Fig. 8 Electron micrograph of free cell adjacent to RPE. The cell possesses finger-like processes similar to the underlying RPE in addition to filopodia typical of macrophages. Pigment granules are contained in a secondary lysosomal complex. $\mathrm{F}=$ Filopodia. $\mathrm{AP}=$ Apical processes. $\mathbf{N}=$ nucleus. $\mathbf{S}=$ Secondary lysosomal complex containing pigment $\times 9600$

(Fig. 8). In the majority of free cells the prominent processes normally seen on the surface of an RPE cell were reduced in number and absent over large areas; however, filopodia were often present. Ultrastructurally such cells appeared half way between a pigment epithelial cell and a free macrophage. This process is shown in schematic form in Fig. 9.
In yet other areas under the detached retina the RPE proliferated to form a layer several cells thick. By light microscopy these cells were seen to be pigmented and spindle-shaped with their long axes parallel to Bruch's membrane (Fig. 10). By electron microscopy it could be seen that the proliferated cells constituted a tight framework, with many cell
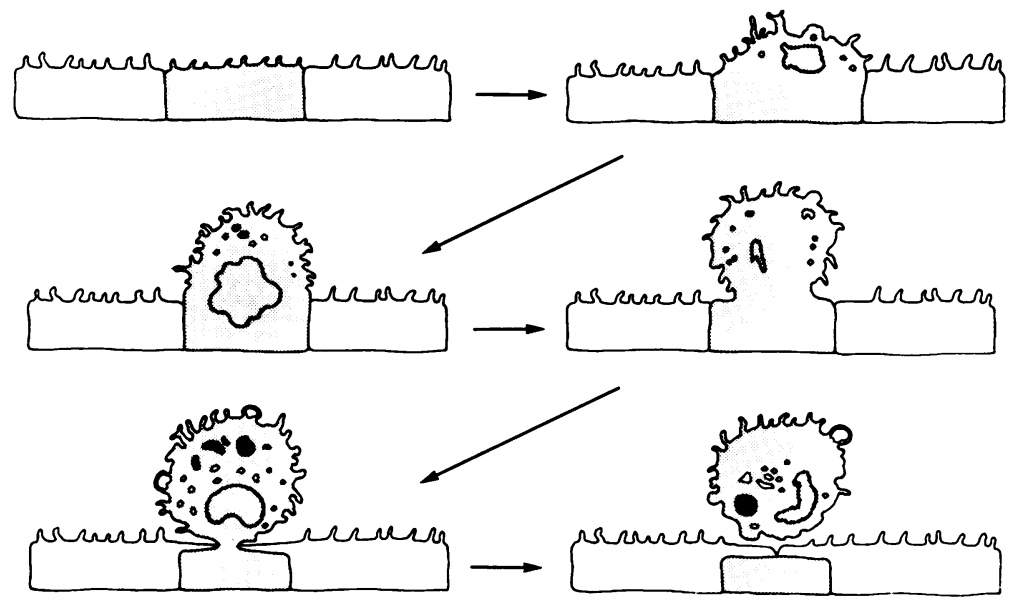

Fig. 9 Diagram of suggested sequence of events leading to the formation of free macrophages from pigment epithelial cells 


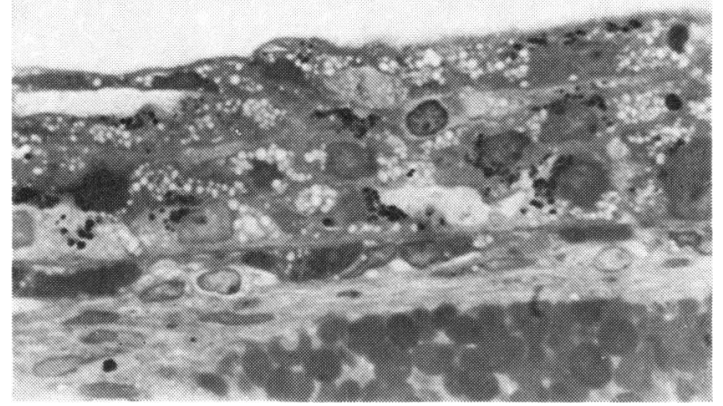

Fig. 10 Light micrograph of RPE underlying detached retina to show an area where $R P E$ cells have proliferated to form a layer several cells thick $\times 650$

junctions between adjacent cells (Fig. 11). The cells adjacent to Bruch's membrane were often irregular in shape, and many appeared to be involved in a similar series of events leading to the formation of free cells as already described. Bruch's membrane was completely covered by RPE, though in places the covering was attenuated. Bruch's membrane underlying the proliferated RPE was considerably thicker than normal (Fig. 12). Among the framework of pigmented cells and in the thickened Bruch's

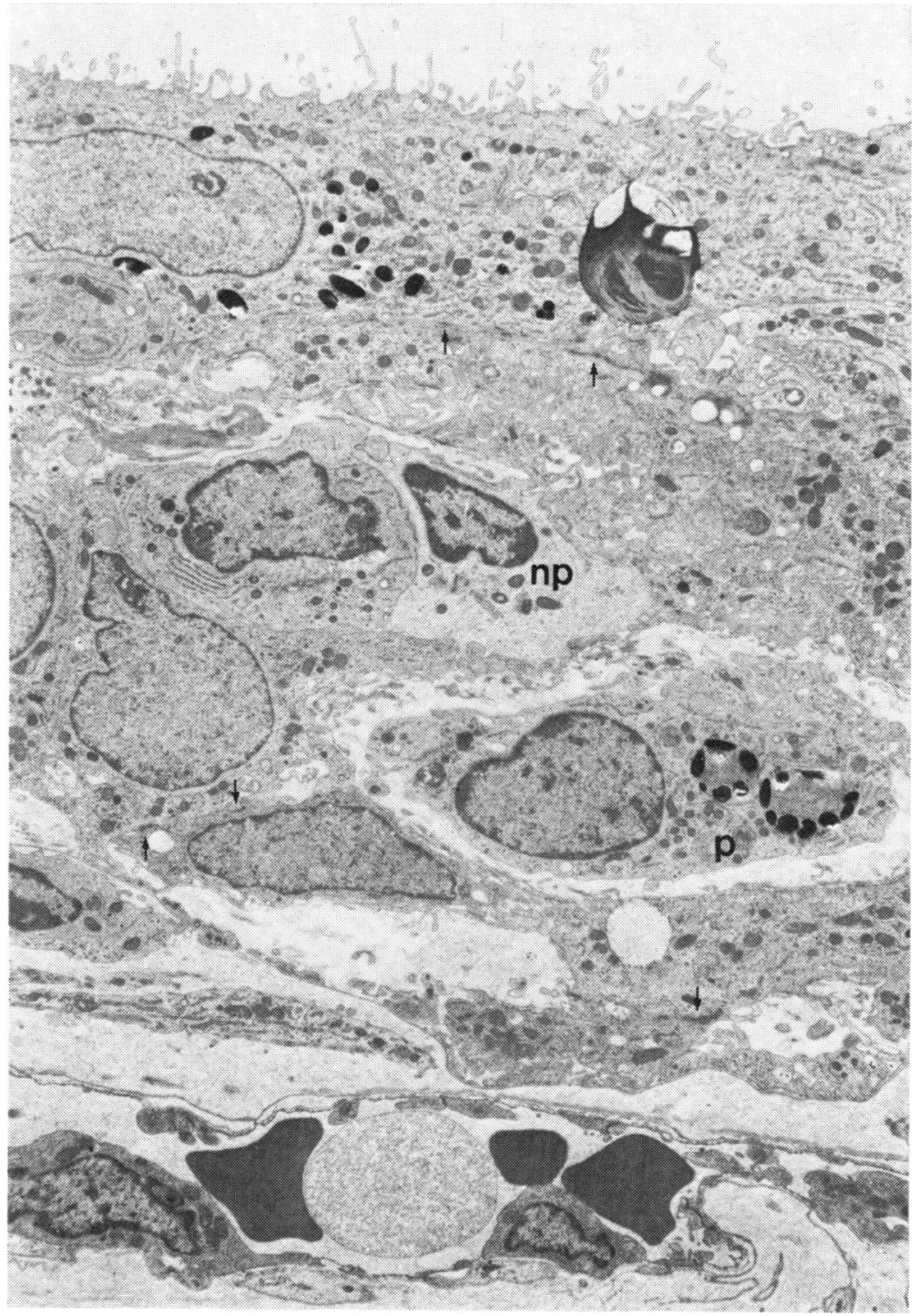

Fig. 11 Electron micrograph of proliferated RPE showing tight framework of cells. Numerous cell junctions occur between the adjacent cells (arrows).

Pigmented (p) and non-pigmented (np). Free cells occur among the framework of proliferated RPE $\times 3040$ 


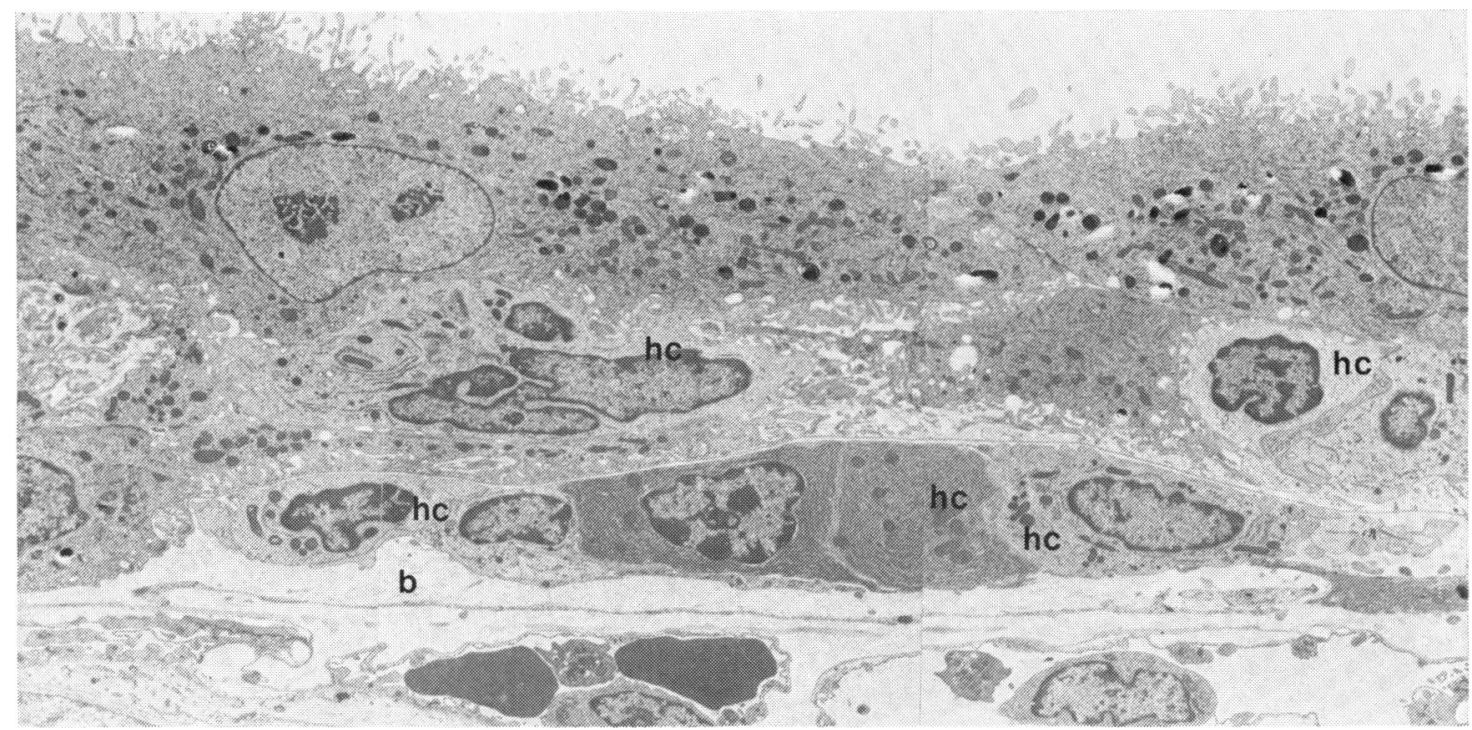

Fig. 12 Electron micrograph of the RPE in an area of RPE proliferation showing thickening of Bruch's membrane (b) and free cells of haematogenous origin (hc) lying in Bruch's membrane or among the RPE cells $\times 2790$

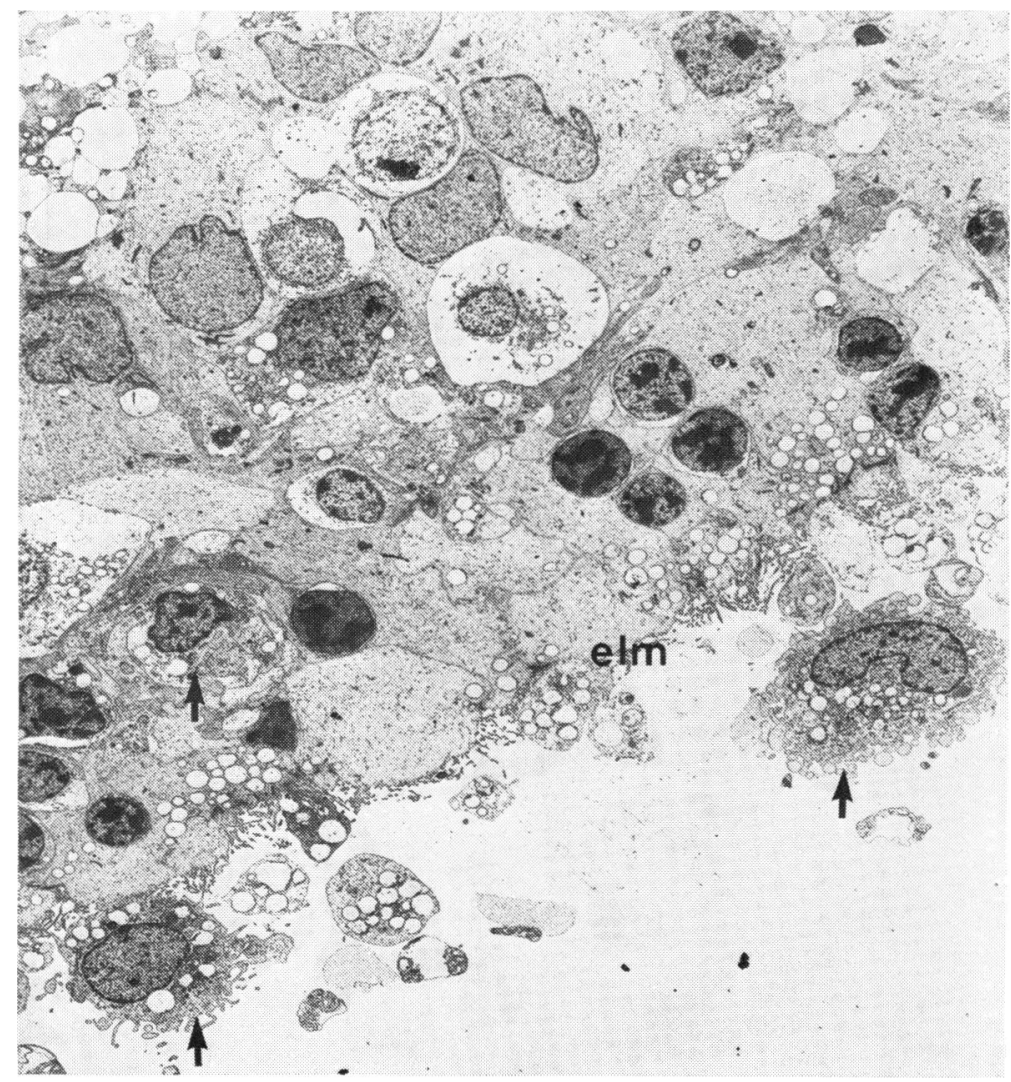

Fig. 13 Electron micrograph $o_{\text {. }}$ outer aspect of detached retina to show macrophage-like cells in the subretinal space underlying the external limiting membrane (elm) and intraretinally (arrows) $\times 1200$ 


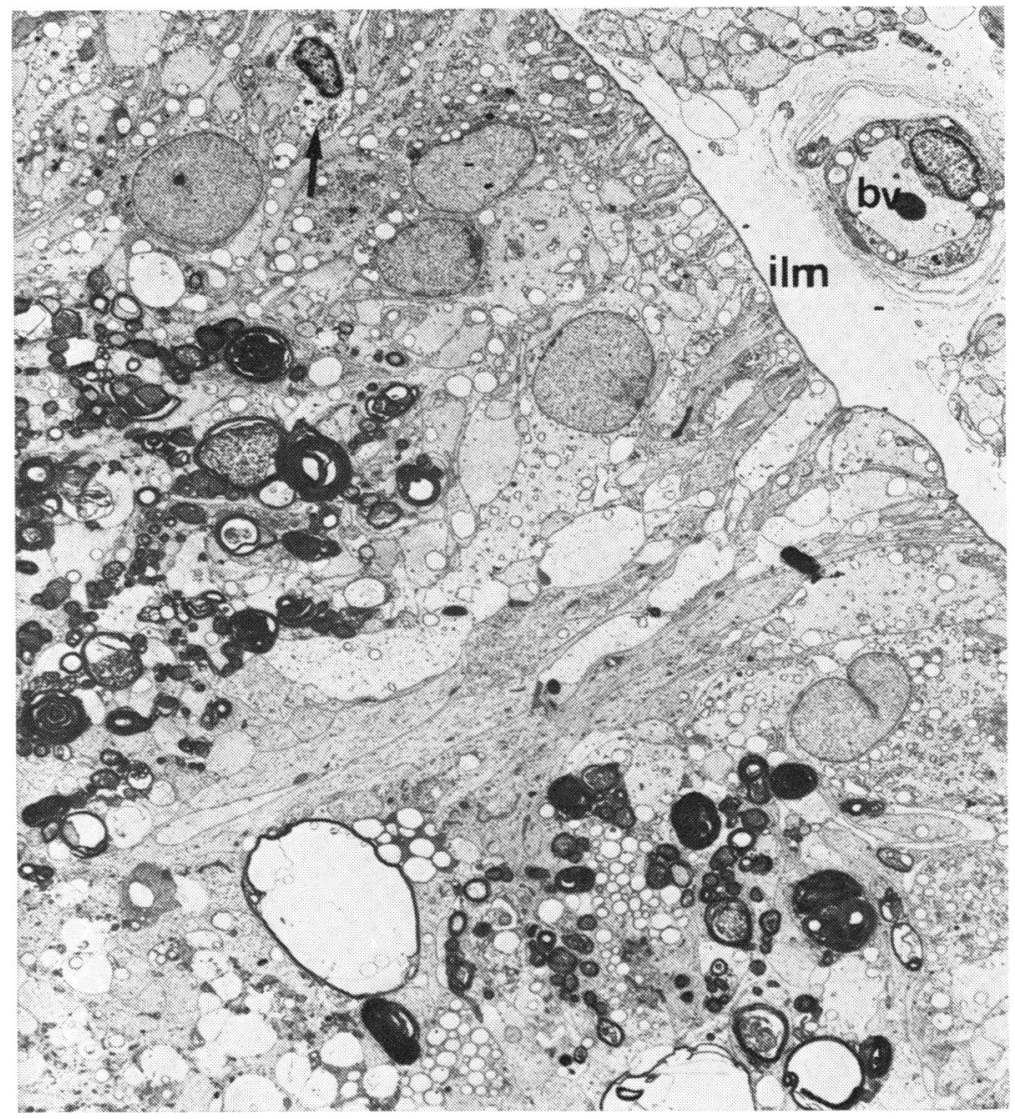

Fig. 14 Inner aspect of detached retina shows normal extraretinal blood vessel (bv), intact internal limiting membrane (ilm), and macrophage-like cells within the retina (arrow) $\times 1200$

membrane free cells were found. Some of these cells contained melanin granules in the cytoplasm or in what appeared to be secondary lysosomal complexes. These cells had many characteristics of the RPE (Fig. 11) and were similar to the free cells already described as probably arising from RPE. The remaining free cells (Figs. 11 and 12) ranged from cells possessing a pale cytoplasm containing few organelles to cells with a more electron-dense cytoplasm containing prominent rough endoplasmic reticulum and Golgi apparatus. Within the thickened Bruch's membrane plasma cells were also found. These were generally located between the basement membrane of the RPE and the endothelium of the choriocapillaris (Fig. 12).

In addition to the changes seen in the RPE layer free macrophage-like cells were found in the subretinal space, underlying the external limiting membrane, and intraretinally (Figs. 13 and 14). These cells were generally without melanin granules and had a cytoplasm of low electron density.

The retinal blood vessels, which in the rabbit are largely superficial to the retina, appeared normal and showed no evidence of cell migration (Fig. 14).

\section{Discussion}

This study has shown that after experimental retinal detachment in the rabbit the RPE undergoes many morphological changes, some of which may represent the dynamic processes by which the RPE may give rise to macrophages which subsequently appear in the subretinal space and within the tissues of the detached retina. In regions where the RPE remains as a monocell layer this process appears to be the major source of retinal and subretinal macrophages.

Other possible origins for these macrophages include the circulating blood, for in areas where the RPE had proliferated to form a multicelled layer various cells of haematogenous origin and others not exactly classifiable could be seen within Bruch's membrane and between the proliferated RPE cells apparently making their way from the choriocapillaris through the RPE layer. Examination of the 
retinal vessels did not suggest migration of macrophages from the retinal capillaries, and it would appear that in areas where there is RPE proliferation Bruch's membrane and the proliferated RPE no longer pose a barrier to the inward migration of blood-borne cells.

Other theoretical sources of macrophages in detached retinae might be the retinal microglia or tissue histiocytes, but no evidence for either of these latter origins was seen in the tissue examined.

An unresolved problem is the fate of the residual cytoplasm of the RPE cell after the vitreal cytoplasmic protrusion has been 'nipped off' by adjacent cells. It was originally thought that the adjacent cells simply squeezed the intervening cells from Bruch's membrane, but on nearly every occasion there appeared to be a residual portion of the cell underlying the extensions of the adjacent cells. The question whether this cell fragment had a nucleus which was out of the plane of section is obviously of interest. The rabbit RPE has a high proportion (up to $80 \%$ ) of binucleate and multinucleate cells (Tso and Friedman, 1967) and it may be that the residual cell fragment is indeed nucleated. No evidence for the further transformation of macrophages to fibroblasts was found in the tissues studied.

\section{References}

Foulds, W. S. (1963). Experimental retinal detachment. Transactions of the Ophthalmological Societies of the United Kingdom, 83, 153-170.

Johnson, N. F., and Foulds, W. S. (1976). The distribution of mucopolysaccharides in normal and detached retinae. Experimental Eye Research, 22, 300-301.

Kroll, A. J. (1969). Secondary retinal detachment. American Journal of Ophthalmology. 68, 223-237.

Kroll, A. J., and Machemer, R. (1968). Experimental retinal detachment in the owl monkey III. American Journal of Ophthalmology, 66, 410-426.

Machemer, R., and Laqua, H. (1975). Pigment epithelium proliferation in retinal detachment. American Journal of Ophthalmology, 80, 1-23.

Mandelcorn, M. S., Machemer, R., Fineberg, E., and Hersch, S. B. (1975). Proliferation and metastasia of intravitreal retinal pigment epithelium cell autotransplants. American Journal of Ophthalmology, 80, 227-237.

Ohkuma, M. (1973). Observations on experimental retinal detachment. Annals of Ophthalmology, 5, 103-116.

Reich-d'Almeida, F. B., and Hockley, D. J. (1975). In situ reactivity of the retinal pigment epithelium I. Experimental Eye Research, 21, 333-345.

Tso, M., and Friedman, E. (1967). The retinal pigment epithelium. Archives of Ophthalmology, 78, 641-653. 